\title{
Obesity status may intervene in the relation between dietary fiber intake and gut microbiota diversity
}

\author{
Noora Houttu, Kati Mokkala and Kirsi Laitinen \\ Institute of Biomedicine, University of Turku, Turku, Finland
}

\section{Abstract}

High diversity is considered a property of gut microbiota that is beneficial for human health. A higher dietary fiber intake has been shown to increase gut microbiota diversity. Similarly, there is preliminary evidence that an excess adiposity relates to low diversity. Our objective was to investigate the relationship between dietary fiber intake and gut microbiota diversity whilst considering the overweight and obesity status. Overweight $\left(n=52\right.$, prepregnancy BMI $\left.\geq 25 \mathrm{~kg} / \mathrm{m}^{2}\right)$ and obese $\left(\mathrm{n}=47, \mathrm{BMI} \geq 30 \mathrm{~kg} / \mathrm{m}^{2}\right)$ women were studied in early pregnancy (a mean of 13 weeks of gestation). The mean daily fiber intake ( $\mathrm{g}$ ) was calculated from 3-day food diaries by computerized software. To evaluate the gut microbiota $\alpha$-diversity we determined the divergence-based measure, phylogenetic diversity (PD), and the qualitative and quantitative species-based measures, Chao 1 and Shannon index, from 16S RNA gene sequencing using QIIME pipeline. Dietary fiber intake (mean \pm SD: $19.7 \pm 6.2 \mathrm{~g}$ ) correlated directly with PD $(\mathrm{rho}=0.33, \mathrm{P}=0.002)$ and Chao 1 index (rho $=0.31, P=0.004)$, but not with Shannon index (rho $=0.20, P=0.06$ ) in all women. Instead, no correlation between BMI and microbiota diversity indexes were seen, except for obese women in whom the BMI correlated inversely with PD (rho $=-0.31, \mathrm{P}=$ 0.04). Interestingly, distinct associations between fiber intake and microbiota diversity were detected in overweight and obese women. Fiber intake correlated directly with $\mathrm{PD}(\mathrm{rho}=0.34, \mathrm{P}=0.02$ vs. rho $=0.32, \mathrm{P}=0.04)$, the measure depicting the phylogenetic branch length, in both overweight and obese women, the correlation with Chao 1 (rho $=0.35, \mathrm{P}=0.02$ ), which depicts the presence or absence of each taxon i.e. richness, was seen only in obese women. Nevertheless, no correlation was seen between fiber intake and Shannon index ( $\mathrm{rho}=-0.004, \mathrm{P}=0.98$ vs. rho $=-0.18, \mathrm{P}=0.25$ ), the measure depicting the presence and absence as well as the abundance of each taxon i.e. richness and evenness, either in overweight or obese pregnant women. We found direct correlations between dietary fiber intake and gut microbiota $\alpha$-diversity but obesity status intervened in this relation. In contrast to overweight women, in obese women, the higher dietary intake was reflected in a higher gut microbiota diversity measured at the level of taxon richness, suggesting that particularly obese pregnant women may benefit from a higher dietary fiber intake.

\section{Conflict of Interest}

There is no conflict of interest 